\title{
Astaxanthin as feed supplement in aquatic animals
}

\begin{abstract}
Astaxanthin is a high value keto-carotenoid pigment renowned for its commercial application in various industries comprising aquaculture, food, cosmetic, nutraceutical and pharmaceutical. Among the verified bio-resources of astaxan-thin are red yeast Phaffia rhodozymaand green alga Haematococcus pluvialis. The supreme antioxidant property of astaxanthin reveals its tremendous potential to offer manifold health benefits among aquatic animals which is a key driving factor triggering the upsurge in global demand for the pigment. Numerous scientific researches devoted over a number of years have persistently demonstrated the instrumental role of astaxanthin in targeting several animal health conditions. This review article evaluates the current best available evidence to judge the beneficial usage of astaxanthin in aquaculture industry. Most apparent is the profound effect on pigmentation, where astaxanthin is frequently utilized as an additive in formulated diets to boost and improve the coloration of many aquatic animal species, and subsequently product quality and price. Moreover, the wide range of other physiological benefits that this biological pigment confers to these animals is also presented which include various improvements in survival, growth performance, reproductive capacity, stress tolerance, disease resistance and immune-related gene expression.
\end{abstract}

Keyword: Astaxanthin; Carotenoid; Disease resistance; Growth; Pigmentation; Reproductive performance 